\title{
A Ruptured Mediastinal Bronchial Artery Aneurysm Treated with Urgent Thoracic Endovascular Aortic Repair
}

\author{
Kazuya Kikutani ${ }^{1}$, Junji Itai $^{1}$, Kohei Ota ${ }^{1}$, Keigo Chosa ${ }^{2}$, \\ Yoshitaka Yamane $^{3}$ and Nobuaki Shime ${ }^{1}$
}

\begin{abstract}
:
Bronchial artery aneurysms (BAA) are a rare but potentially life-threatening complications because of the massive hemothorax or hemoptysis that occurs with ruptures. A 79-year-old woman was transferred to our hospital because of the sudden onset of back pain, syncope, and subsequent hypotension. Computed tomography showed a left BAA with bilateral hemothorax and hemomediastinum. Transcatheter bronchial artery embolization failed because of the anatomical location, and she went into cardiopulmonary arrest. Cardiopulmonary resuscitation was performed with successful revival. Urgent thoracic endovascular aortic repair to cover the root of the left bronchial artery was successful, and she survived without any neurological deficits.
\end{abstract}

Key words: bronchial artery, aneurysm, rupture, stent graft, thoracic endovascular aortic repair

(Intern Med 59: 1283-1286, 2020)

(DOI: 10.2169/internalmedicine.4004-19)

\section{Introduction}

Bronchial artery aneurysm (BAA) is a rare disease, but it can cause severely disturbed hemodynamics when it ruptures (1). Conventionally, transcatheter bronchial artery embolization (TAE) or open chest surgery is conducted to isolate the aneurysm.

We herein report a case of successful treatment of a ruptured mediastinal BAA that required urgent thoracic endovascular aortic repair under conditions of severely disturbed hemodynamics.

\section{Case Report}

A 79-year-old woman with Sjögren's syndrome was admitted to a clinic because of the sudden onset of back pain with subsequent syncope. During a medical examination conducted by a previous doctor, she developed hypotension and tachycardia. She was transferred to our emergency and critical care center after the confirmation of mediastinal hematoma and bilateral hemothorax as shown by enhanced computed tomography (CT).

Upon arrival at our hospital, her blood pressure was 53/33 $\mathrm{mmHg}$, and her heart rate was $140 \mathrm{bpm}$ under dopamine infusion $(10 \mu \mathrm{g} / \mathrm{kg} / \mathrm{min})$. Her respiratory rate was 24 breaths per minute, and her percutaneous oxygen saturation was not measurable because of peripheral circulatory failure. Her Glasgow Coma Scale score at admission was 13 points (E3 V4M6). She complained of back pain. A blood examination showed a hemoglobin level of $6.8 \mathrm{~g} / \mathrm{dL}$, and the platelet count was $129,000 / \mu \mathrm{L}$. An arterial blood gas analysis showed an elevated lactate level $(4.3 \mathrm{mmol} / \mathrm{L})$. We immediately initiated blood transfusion, and her hemodynamics transiently stabilized. Enhanced CT showed a mediastinal hematoma, bilateral hemothorax, and a 9-mm diameter aneurysm of the left bronchial artery (Fig. 1) beside the descending aorta. The inflow vessel was $2 \mathrm{~mm}$ in diameter and $4 \mathrm{~mm}$ in length. Extravasation was not confirmed. We diagnosed her with hemorrhagic shock due to bilateral hemothorax and hemomediastinum caused by mediastinal rupture of the BAA.

We originally attempted TAE. Bronchial angiography confirmed the BAA with extravasation (Fig. 2). Embolization of

\footnotetext{
${ }^{1}$ Department of Emergency and Critical Care Medicine, Graduate School of Biomedical \& Health Sciences, Hiroshima University, Japan, ${ }^{2}$ Department of Diagnostic Radiology, Hiroshima University, Japan and ${ }^{3}$ Department of Cardiovascular Surgery, Hiroshima University School of Medicine, Japan

Received: October 4, 2019; Accepted: December 24, 2019; Advance Publication by J-STAGE: February 19, 2020

Correspondence to Dr. Nobuaki Shime, nshime@hiroshima-u.ac.jp
} 
(A)

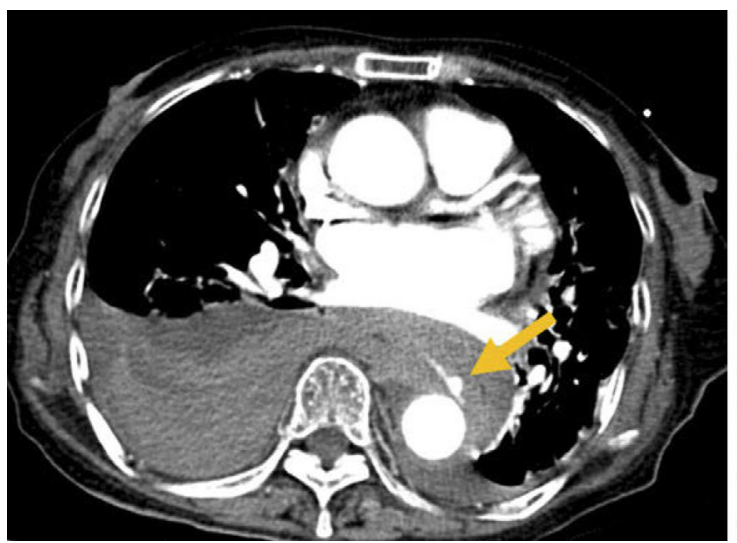

(B)

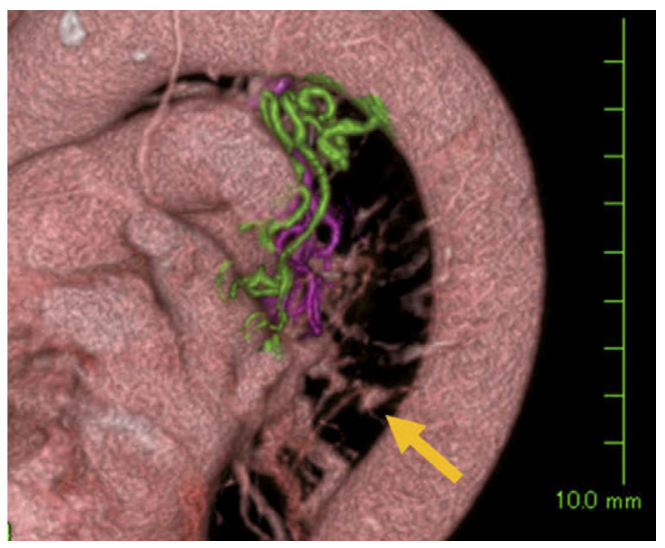

Figure 1. An enhanced computed tomography scan shows a mediastinal hematoma and bilateral hemothorax, especially on the right side (A). A bronchial artery aneurysm (arrow) is present near the descending aorta as shown by an enhanced computed tomography scan (A) and (B) by three-dimensional reconstruction of the enhanced computed tomography scan.

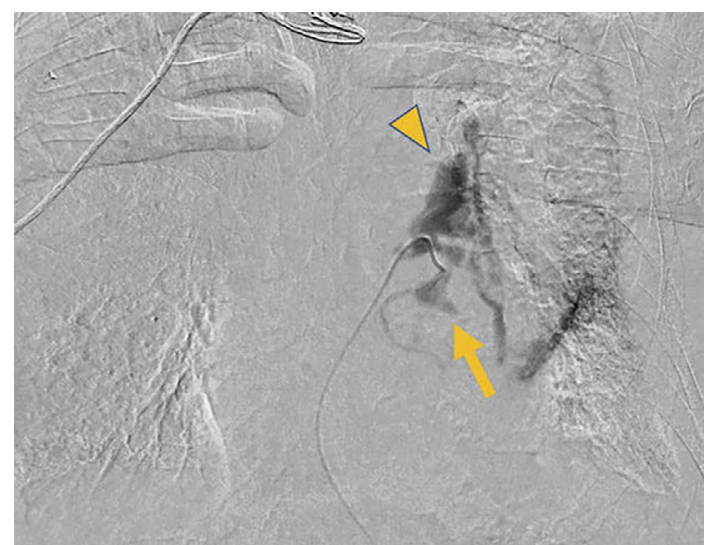

Figure 2. Selective bronchial angiography shows a bronchial artery aneurysm (arrow) and extravasation of contrast medium (arrowheads).

the inflow vessel failed because the aneurysm was too close to the descending aorta. We then chose to perform thoracic endovascular aortic repair (TEVAR). However, during preparation for TEVAR, her blood pressure dropped, and we performed intra-aortic balloon occlusion (IABO) to control the bleeding. In addition, we attempted to embolize the outflow vessel during TEVAR preparation. However, selective catheterization into the left bronchial artery was not possible because the catheter was diverted while performing IABO positioning. Thereafter, she went into cardiopulmonary arrest. We were thus unable to embolize the outflow vessel. Cardiopulmonary resuscitation and massive transfusion in combination with IABO to control the bleeding were immediately performed. The patient's spontaneous circulation recovered after a few minutes. This was followed by successful urgent insertion of a stent graft (Gore $\mathrm{TAG}^{\circledR}$; W. L. Gore \& Associates, Flagstaff, USA) in the descending aorta to cover the root of the left bronchial artery. Complete isolation of the BAA and the absence of endoleaks and collateral circulation were confirmed by aortography (Fig. 3), as well as by selective artery angiography of the right bronchial artery, right supreme intercostal artery, and upper lobe branch of the left bronchial artery.

Postoperatively, her anemia and hemothorax were well controlled, and she was extubated on day 4. Postoperative CT showed complete isolation of the BAA. On day 11, she was transferred to another hospital for rehabilitation without any neurological deficits.

\section{Discussion}

$\mathrm{BAA}$ is a rare disease, presenting in only $1 \%$ of those who undergo selective bronchial angiography (2). The etiology of BAAs is uncertain, but an increased blood flow of the bronchial artery might contribute to the formation of an aneurysm $(1,3)$. Bronchiectasis, hypertension, arteriosclerosis, chronic obstructive pulmonary disease, tuberculosis, and trauma have been reported as typical risk factors for BAA (1-3). In our case, a lung CT scan on admission showed bronchiectasis as a possible cause of the BAA.

Although BAAs are often subclinical and detected incidentally on CT, sudden rupture can occur with various symptoms, depending on their location $(2,3)$. Mediastinal BAA rupture causes hemomediastinum or symptoms mimicking aortic dissection (e.g., chest pain, back pain), whereas intrapulmonary BAA rupture is often associated with hemoptysis (3). Dysphagia, cough, and hoarseness are also found in patients with BAA $(2,4)$. In our case, the patient presented with the sudden onset of back pain, syncope, severe hypotension, bilateral hemothorax, and hemomediastinum. These findings are in line with possible symptoms of mediastinal BAA rupture.

Conventional treatment for a ruptured BAA includes open chest surgery or TAE. TAE is often the first choice for a ruptured BAA because of its low invasiveness, although it is 
(A)

(B)

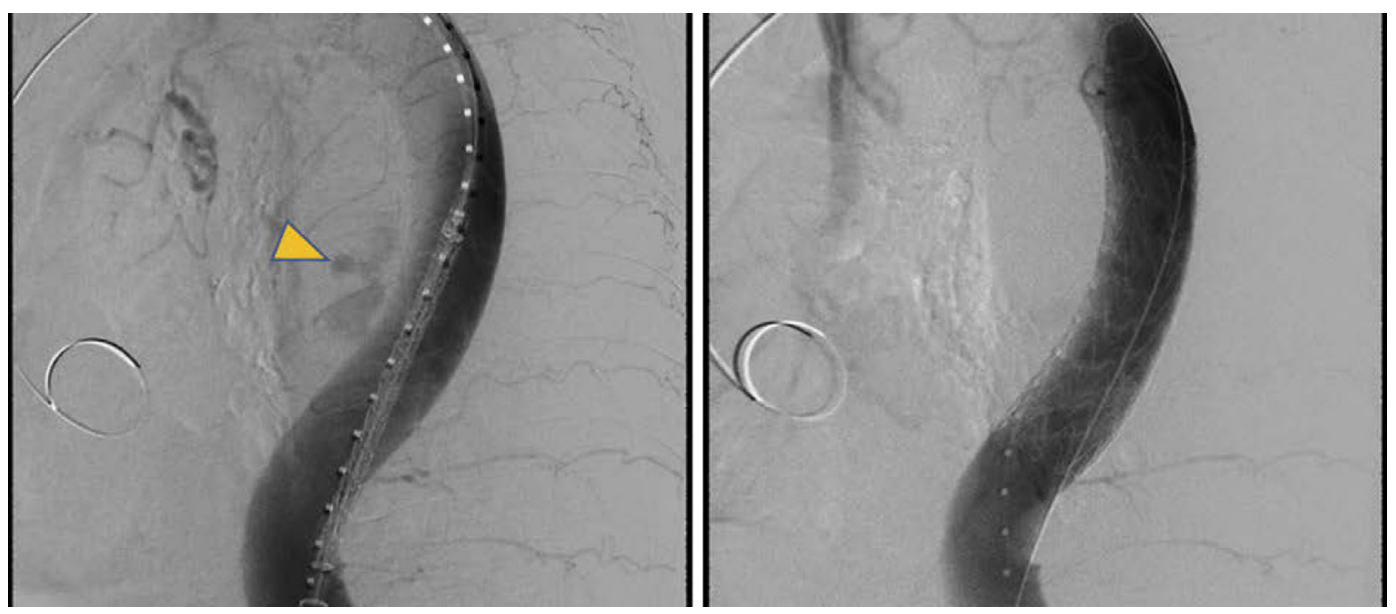

Figure 3. Extravasation of the contrast medium before (A, arrowheads) and after (B) completion of TEVAR.

Table. Reports of Bronchial Artery Aneurysms Treated via Thoracic Endovascular Aortic Repair Aortic Repair.

\begin{tabular}{lccccccc}
\hline Case & Age $(\mathrm{y})$ & Sex & Symptom & Treatment & Rupture & Shock & Size $(\mathrm{mm})$ \\
\hline 1 (ref3) & 72 & $\mathrm{M}$ & None & TEVAR+TAE & No & No & 25 \\
$2($ ref4) & 79 & M & Hoarseness & TEVAR & No & No & $60 \times 55$ \\
$3($ ref5) & 76 & F & None & TEVAR+TAE & No & No & 40 \\
$4($ ref6) & 69 & F & Dysphagia & TEVAR+TAE & No & No & 40 \\
$5($ ref7) & 74 & M & None & TEVAR+TAE & No & No & 60 \\
$6($ ref8) & 67 & M & Chest pain & TEVAR+TAE & Yes & No & $40 \times 30$ \\
& & & Back pain & & & & \\
$7($ ref9) & 66 & M & Dysphagia & TEVAR+TAE & No & No & 40 \\
$8($ ref10) & 64 & M & None & TEVAR & No & No & 18 \\
$9($ ref11) & 73 & F & Hemoptysis & TEVAR+TAE & No & No & 32 \\
$10($ ref12) & 77 & M & None & TEVAR+TAE & No & No & 53 \\
$11($ ref13) & 89 & M & None & TEVAR & No & No & 38 \\
$12($ ref14) & 72 & F & None & TEVAR & No & No & 30 \\
$13($ ref15) & 23 & M & Hemoptysis & TEVAR+TAE & Yes & No & 28 \\
Our case & 79 & F & Back pain & TEVAR & Yes & Yes & 9 \\
\hline
\end{tabular}

M: male, F: female, TEVAR: thoracic endovascular aortic repair, TAE: transcatheter bronchial artery embolization

The aneurysm in each reported case was located in a mediastinal position.

sometimes difficult when the aneurysm has a short neck. In such cases, previous studies reported the successful treatment effects of a stent graft for BAA (3-15) (Table). However, most of the treatments in these previous studies were indicated for an unruptured BAA, while only two reports indicated a ruptured $\operatorname{BAA}(8,15)$. Our patient is the first to have undergone urgent TEVAR for a ruptured BAA while performing cardiopulmonary resuscitation. Preparation of TEVAR simultaneously with the indication for TAE by anticipating the difficulty of TAE based on enhanced CT should be considered in this situation.

A combination of TAE to embolize the outflow branch and TEVAR to embolize the inflow branch should be considered because TEVAR alone cannot embolize the outflow branch, resulting in a retrograde flow. However, in our case, we fortunately confirmed no retrograde flow after TEVAR. Because the pulmonary artery is a low-pressure system, embolization of the outflow may not always be necessary $(7,14)$. The findings from our case suggest that embolization of the inflow artery with TEVAR alone is relatively non-time-consuming compared with embolizing both the outflow and inflow branches with TAE and may help save a patient's life. This strategy might be useful in urgent situations, such as hemorrhagic shock due to aneurysm rupture.

If our patient's hemodynamics had not stabilized after performing TEVAR, we would have performed selective artery angiography to intervene in the collateral circulation of 
the bronchial aneurysm. Furthermore, if bleeding still persisted, we would have approached through the pulmonary artery to evaluate the collateral circulation. If all of these procedures were performed and the bleeding continued, open chest surgery might have been the final option.

\section{Conclusion}

We present a case of successful treatment of a ruptured mediastinal BAA with urgent TEVAR.

The authors state that they have no Conflict of Interest (COI).

\section{Acknowledgement}

We thank Ellen Knapp, PhD for editing a draft of this manuscript.

\section{References}

1. Di X, Ji DH, Chen Y, Liu CW, Liu B, Yang J. Endovascular treatment of ectopic bronchial artery aneurysm with brachiocephalic artery stent placement and coil embolization: a case report and literature review. Medicine (Baltimore) 95: e4461, 2016.

2. Lü PH, Wang LF, Su YS, et al. Endovascular therapy of bronchial artery aneurysm: five cases with six aneurysms. Cardiovasc Intervent Radiol 34: 508-512, 2011.

3. Sakai T, Razavi MK, Semba CP, Kee ST, Sze DY, Dake MD. Percutaneous treatment of bronchial artery aneurysm with use of transcatheter coil embolization and thoracic aortic stent-graft placement. J Vasc Interv Radiol 9: 1025-1028, 1998.

4. Kasashima F, Endo M, Kosugi I, et al. Mediastinal bronchial artery aneurysm treated with a stent-graft. J Endovasc Ther 10: 381385, 2003.

5. Tsolaki E, Salviato E, Coen M, Galeotti R, Mascoli F. Double right bronchial artery aneurysm treated with combined procedures. Eur J Vasc Endovasc Surg 34: 537-539, 2007.

6. Sanchez E, Alados P, Zurera L, et al. Bronchial artery aneurysm treated with aortic stent graft and fibrin sealant. Ann Thorac Surg 83: 693-695, 2007.

7. Takahashi Y, Tsutsumi Y, Monta O, et al. Stent grafting for giant bronchial artery aneurysm disguised as an aneurysm with multiple feeding arteries. Ann Thorac Surg 89: 1652-1654, 2010.

8. Hu CX, Huang S, Xu ZW, Chen W, Huang JS, Fu Z. Combination of aortic stent-graft and arterial embolization for ruptured bronchial artery aneurysm. Ann Thorac Surg 92: e19-e21, 2011.

9. Guzzardi G, Cerini P, Fossaceca R, Commodo M, Micalizzi E, Carriero A. Endovascular treatment of bronchial artery aneurysm with aortic stent-graft placement and coil embolization. Ann Vasc Surg 26: 1013.e5-1013.e8, 2012.

10. Arici V, Bozzani A, Odero A. Successful endovascular treatment of a bronchial artery aneurysm refractory to transcatheter embolization. Ann Vasc Surg 27: 802.e13-802.e15, 2013.

11. Kim YK, Sung YM, Kim JH, Byun SS, Park YN, Lee SP. Aortic stent-graft for a giant bronchial artery aneurysm with ultrashort neck. Ann Thorac Cardiovasc Surg (Suppl): 781-785, 2014.

12. Samura M, Morikage N, Yamashita O, Murakami M, Suehiro K, Hamano K. Combination of aortic stent grafting and arterial embolization for bronchial artery aneurysm associated with bronchialpulmonary arterial fistula. J Vasc Interv Radiol 26: 1077-1079, 2015.

13. Fiorucci B, Simonte G. Treatment of a bronchial artery aneurysm with placement of an aortic thoracic stent-graft. Eur J Vasc Endovasc Surg 51: 717, 2016.

14. Matsumoto $T$, Uchida $T$, Ono $T$, et al. Bronchial artery aneurysm treated using aortic stent graft alone: a case report. Ann Vasc Dis 10: 152-154, 2017.

15. Wang J, Zhou G, Liang B, et al. Combination of aortic stent-graft and arterial embolization for multiple bronchial artery aneurysms associated with diffuse bronchial-pulmonary arterial fistulas. J Vasc Interv Radiol 29: 1283-1285, 2018.

The Internal Medicine is an Open Access journal distributed under the Creative Commons Attribution-NonCommercial-NoDerivatives 4.0 International License. To view the details of this license, please visit (https://creativecommons.org/licenses/ by-nc-nd/4.0/).

(C) 2020 The Japanese Society of Internal Medicine Intern Med 59: 1283-1286, 2020 